\title{
Felodipine loaded PLGA nanoparticles: preparation, physicochemical characterization and in vivo toxicity study
}

Utpal Jana ${ }^{*}$, Anjan Kumar Mohanty, Sovan Lal Pal, Prabal Kumar Manna and Guru Prasad Mohanta

\begin{abstract}
Felodipine, a calcium channel blocker has been widely used for the treatment of hypertension and cardiovascular diseases; but the frequent dosing is needed for its poor solubility and variable bioavailability. In present study an attempt has been made to overcome the problems through nanoparticulate delivery system using poly ( $D$, L-lactic-co-glycolic acid) polymer keeping in the view to get better sustainability of the formulation. The nanoparticles were prepared by single emulsion solvent evaporation technique and the physico-chemical characterization of prepared nanoparticles confirmed the particles were nanosize range with smooth and spherical morphology. Further, the compatibility of drug-polymer combination was analyzed by FTIR and DSC study. The in vitro drug release study of PLGA nanoparticles showed longer duration of drug release with reduced burst release compared with pure felodipine. The in vitro drug release data were fitted with various mathematical models to establish the drug release mechanism from the nanoparticles and found to follow mixed order kinetics. The in vivo toxicity study in albino mice showed no noticeable change in biochemical parameters and histopathology of organs. Hence, the developed felodipine nanoparticles were prepared, characterized and could possibly be advantageous for prolonged drug release and improving the antihypertensive effect.
\end{abstract}

Keywords: Felodipine; PLGA; Release kinetics; Biochemical parameter; Nanoparticles

\section{Background}

Hypertension is considered to be a major risk factor in the development of cardiovascular diseases (CVD), a leading cause of death worldwide. Hypertension is common in both developed and low- and middle-income countries [1]. The recent study reveals that half of the treated patients are not able to achieve desired blood pressure targets $[2,3]$. It has become a challenge to maintain the blood pressure for long time that can significantly reduce the risk of morbidity and mortality [4]. There are number of medicines used for the treatment of high blood pressure and patients might need to take their medicine for several years.

Calcium channel blockers (CCBs) are broadly used for the treatment of systemic arterial hypertension. The function of CCBs is to inhibit voltage dependent L- type calcium channels in vascular smooth muscle and heart.

* Correspondence: jana.utpal@gmail.com

Department of Pharmacy, Annamalai University, Annamalai Nagar, Tamil Nadu, India
The antihypertensive effect of CCBs is due to arterial dilatation that causes smooth muscle relaxation. Felodipine, a dihydropyridine calcium channel blocker, has been widely used for the treatment of hypertension [4]. Clinical study revealed that felodipine has dose dependant activity for reduction of blood pressure and has antihypertensive activity similar to other antihypertensive drugs including beta blockers and angiotensin-converting enzyme (ACE) inhibitors. However, the felodipine is extensively metabolised in the gut and liver and is excreted almost entirely as metabolites [4]. Felodipine is a lipophilic crystalline powder and practically insoluble (BCS Class-II) in water (solubility-0.5 mg/l) [4]. As felodipine has poor and variable bioavailability, the multiple daily dosing is needed. The multiple daily dosing not only has the issues with fluctuation of plasma drug concentration but may also lead to poor patients' adherence.

The bioavailability of poorly water soluble drugs is dissolution rate dependant. In order to improve the bioavailability various approaches were suggested, including

\section{实}


solubilisation, use of co-solvents, salt formation, micronization and complexation with cyclodextrins $[5,6]$. However, more amounts of additives are required for these techniques limiting their use from the safety perspective. Another effective way to enhance the bioavailability of the drug is by making the particle size within the nano-meter range. The reduced particle size into nanometre size leads to improve dissolution rate because of increased surface area [7]. The suitable stabilizing agents are used to prevent the nano-sized particle aggregation. However, if the drug is coated with suitable polymer the crystalline nature of the drug can possibly be prevented and can be stabilized over long period of time.

In recent years, biodegradable polymeric nanoparticles have been used to improve the solubility and dissolution rate of both hydrophilic and hydrophobic drugs. The nanoparticles attracted considerable attention as potential drug delivery devices in view of their applications as sustained release of drugs, their ability to target particular organs/tissues, which helps in sustained oral drug delivery beneficial for the patients $[8,9]$. The sub-cellular size of the nanoparticles can improve the stability and also allows relatively higher intracellular uptake of drug than other particulate systems $[10,11]$. The widely used methods for nanoparticles preparation are solvent evaporation, high pressure homogenization [12], nano-precipitation and emulsion diffusion etc. $[13,14]$.

Poly (D, L-lactic-co-glycolic acid) or PLGA is one of the most popular biodegradable and non toxic polymers used for preparation of microparticles and nanoparticles $[15,16]$. PLGA is randomly hydrolyzed into their biocompatible metabolite, lactic acid and glycolic acid and eliminated from the body as carbon dioxide and water [15]. PLGA is widely used for the manufacture of implants, internal sutures and also used for controlled and targeted drug delivery systems [16]. The changes in tissue distribution and pharmacokinetic profile were observed, when the drug was incorporated into PLGA nanoparticulate system. As the particle sizes of the nanoparticles are small enough they are expected to reach angiogenetic area and circulate through the capillaries $[15,16]$. These may lead to improve therapeutic efficacy and patient compliance. In the present study, felodipine loaded PLGA nanoparticles were prepared, characterized and evaluated for in vivo toxicity study in animal model.

\section{Methods}

\subsection{Materials}

Felodipine was a kind gift from Cadila Healthcare Limited (Ahmedabad, India). Poly (D, L-lactic-co-glycolic) acid (PLGA 50:50) $(\mathrm{Mw}=18,000)$ was procured from Sigma Aldrich. Lutrol ${ }^{\circ}$ F-68 (Poloxamer 188) was purchased from SD Fine Chemicals. Distilled- deionized water was prepared with Milli-Q plus System (Elix 10, Millipore corp. India). All other chemicals used were of the highest available grade.

\subsection{Preparation of drug loaded PLGA nanoparticles}

The felodipine loaded nanoparticles were prepared by single emulsion solvent evaporation in accordance with method reported with slight modification [17]. Briefly, $100 \mathrm{mg}$ of PLGA (copolymer ratio 50:50) polymer and weighed quantity of felodipine (equivalent to $20 \% \mathrm{w} / \mathrm{w}$ dry weight of polymers) was dissolved in acetone at room temperature to make clear solution. The solution was mixed with $25 \mathrm{ml}$ of aqueous phase containing 1\% $(\mathrm{w} / \mathrm{v})$ of poloxamer-188 with a constant flow rate of $1 \mathrm{ml} / \mathrm{min}$. The mixture was then homogenized using a probe homogenizer (VIRTIS, Cyclone IQ, USA), at constant agitation speeds of $15000 \mathrm{rpm}$ in an ice bath to form oil-in-water $(\mathrm{O} / \mathrm{W})$ emulsion. Then the emulsion was kept at room temperature under gentle stirring for $24 \mathrm{~h}$ to evaporate the organic solvent. The prepared nanosuspension was then centrifuged at 30,000 rpm, for 15 min (Sorvall Ultracentrifuge, USA). The pellets were collected and washed with double distilled water to remove any unbound poloxamer-188 and free drugs. Finally, the pallets were freeze-dried (Freezone 6lt, Labconco Corp., $\mathrm{MO}$ ) to get powdered nanoparticles and kept in freeze for further use.

\subsection{Physico-chemical characterization of nanoparticles 2.3.1 Particle size and zeta potential}

Dynamic laser scattering (DLS) was used to measure the hydrodynamic diameter (d $\mathrm{nm})$ and Laser Doppler Anemometry (LDA) was used to determine zeta potential $(\mathrm{mV})$. The DLS and LDA were analyzed using Zetasizer 3000 (Malvern Instruments, Malvern, UK). The particle size and zeta potential was determined by using a dilute suspension of nanoparticles $(100 \mu \mathrm{g} / \mathrm{ml})$ prepared in double distilled water and sonicated on an ice bath for 30 seconds. For each batch of sample, the mean diameter \pm standard deviations of three determinations were calculated under identical conditions. The polydispersity index (PDI) was also measured to determine particle size distribution.

\subsubsection{Estimation of drug entrapment efficiency and loading capacity by RP-HPLC method}

The entrapment efficiency (EE) and loading capacity (LC) of felodipine loaded nanoparticles were measured by reverse phase High Performance Liquid Chromatography (RP-HPLC) method [18] with slight modification. Briefly, $1 \mathrm{mg} / \mathrm{ml}$ drug loaded nanoparticle solution was prepared in methanol and $20 \mu \mathrm{L}$ of the sample was injected manually to HPLC equipped with Shimadzu LC-20 AD PLC pump and SPD-M20A PDA detector. The output signal was monitored and integrated using Shimadzu CLASSVP Version 6.12 SP1 software. The chromatographic 
separation was carried out using analytical column Phenomenex C18 $(150 \times 4.6 \mathrm{~mm}, 5 \mu)$. The measurements were made at $240 \mathrm{~nm}$ maintaining the flow rate at $1.0 \mathrm{~mL} / \mathrm{min}$ and ambient condition using thermostat. The amount of the drug present in the sample was determined from the peak area of the chromatogram correlated with the standard curve.

\subsubsection{Fourier transforms infrared spectroscopy (FTIR)}

Infrared spectroscopy study was carried out using FT-IR spectrophotometer (Perkin Elmer, FT-IR Spectrometer, SPECTRUM RX I, USA) and the spectrum was recorded in the range of 4000-400 $\mathrm{cm}^{-1}$ with resolution of $2 \mathrm{~cm}^{-1}$. Samples were mixed separately with potassium bromide (200-400 mg) and compressed by applying pressure of $200 \mathrm{~kg} / \mathrm{cm}^{2}$ for $2 \mathrm{~min}$ in hydraulic press to prepare the pellets. The pellets of the native drug, polymer and the nanoparticles were analysed by placing it on the light path and the spectrum was obtained.

\subsubsection{Differential scanning calorimetry (DSC)}

DSC is a thermo-analytical technique used to observe fusion and crystallization events of the drug in the prepared nanoparticles. Thermogram was obtained by the DSC analysis (DSC-60, Shimadzu, Japan). Approximately, weighed $2 \mathrm{mg}$ of native drug, polymer and nanoparticles were placed separately into sealed standard aluminium pan and scanned between $25^{\circ} \mathrm{C}$ to $300^{\circ} \mathrm{C}$ with heating rate of $10^{\circ} \mathrm{C} /$ minute under nitrogen atmosphere. An empty aluminium pan considered as reference.

\subsubsection{Scanning electron microscopy (SEM)}

The shape and surface morphology of prepared nanoparticles were examined by scanning electron microscopy (SEM) (ZEISS EVO18, Carl Zeiss SMT GmbH, Germany). Moisture free lyophilised powdered samples were consigned on aluminium stubs using adhesive tapes and coated with gold using sputter coater and photomicrographs were taken at an acceleration voltage of $10-30 \mathrm{kV}$.

\subsubsection{Atomic force microscopy (AFM)}

Atomic force microscopy (AFM) studies were carried out to characterize the surface morphology of the drug loaded nanoparticles. The suspension of freeze dried powder was prepared with milli-Q water and dried in air on a clean glass surface for overnight. The observation was performed with AFM (JPK NanoWizard II, JPK instrument, Berlin, Germany) with silicon probes with pyramidal cantilever having force constant of $0.2 \mathrm{~N} / \mathrm{m}$. The scan speed of $2 \mathrm{~Hz}$ and $312 \mathrm{kHz}$ resonant frequency was used to obtain the images [19].

\subsubsection{In vitro drug release study and release kinetics}

The in vitro drug release study was carried out by using rotating basket method with some modification [20,21]. The felodipine loaded nanoparticles (containing $5 \mathrm{mg}$ felodipine) were suspended in glass bottles containing $100 \mathrm{ml}$ of phosphate buffer $\mathrm{pH}$ 6.8. Glass bottles were placed in beaker and kept in incubator shaker (50 rpm) throughout the study, with temperature adjusted to $37^{\circ} \mathrm{C}$. At specified time interval $10 \mathrm{ml}$ samples were withdrawn and centrifuged at $12,000 \mathrm{rpm}$ for $30 \mathrm{~min}$. The precipitates were resuspended with $10 \mathrm{ml}$ of fresh phosphate buffer and added to the glass bottle and supernatants were collected and analyzed by RP-HPLC. All the measurements were carried out in triplicate.

The drug release mechanism from the nanoparticulate systems were analyzed by various mathematical models. The drug release data were fitted with mathematical models including zero order kinetic [Eq. (1)], first order kinetic [Eq. (2)], Higuchi kinetic [Eq. (3)] and KorsmeyerPeppas model [Eq. (4)].

$$
\begin{aligned}
& Q_{t}=K_{0} t \\
& \ln Q_{t}=\ln Q_{t}-K_{1} t \\
& Q_{t}=K_{h} t^{1 / 2} \\
& M_{t} / M_{\alpha}=K_{p} t^{n}
\end{aligned}
$$

The plots were made: $\mathrm{Q}_{\mathrm{t}}$ vs. $\mathrm{t}$ (zero order kinetic), ln $\left(Q_{0}-Q_{t}\right)$ vs. $t$ (first order kinetic) and $Q_{t}$ vs. $t^{1 / 2}$ (Higuchi model), where $Q_{t}$ is the percentage of drug release at time $t, Q_{0}$ is the initial amount of drug present in the formulation and $\mathrm{K}_{0}, \mathrm{~K}_{\mathrm{t}}$ and $\mathrm{K}_{\mathrm{h}}$ are the constants of the equations. The first $60 \%$ drug release was fitted in Korsmeyer-Peppas model, where $M_{t} / M_{\alpha}$ are the fraction of drug release at time $t, K_{p}$ is the rate constant and " $n$ " is the release exponent. The value of " $n$ " is calculated from the slop of the plot of $\log$ of fraction of drug released $\left(M_{t} / M_{\alpha}\right)$ vs. $\log$ of time to characterize the different release mechanism $[20,21]$.

\subsection{In vivo studies}

\subsubsection{Animals}

Wistar albino mice were procured from Central Animal House, Rajah Muthiah Medical College, Annamalai University, India and housed in the Institutional animal house under standard environmental conditions $\left(22 \pm 3^{\circ} \mathrm{C}\right.$, $55 \pm 5 \%$ humidity and $12 \mathrm{~h} / 12 \mathrm{~h}$ dark/light cycle) and maintained with free access to standard diet and water ad libitum. All animal experimentations were executed in compliance with the guidelines of the Committee for the Purpose of Control and Supervision of Experimental Animals (CPCSEA), New Delhi, Government of India. 


\subsubsection{Acute oral toxicity study in mice}

The animals were randomly selected and assigned to following four test groups (6 mice in each group) namely Group I (Control groups, treated with normal saline), Group II, III, IV and V (Test groups, treated with felodipine loaded nanoparticles equivalent to 60, 120, 240 and $480 \mathrm{mg} / \mathrm{kg} \mathrm{BW}$ of felodipine in distilled water). The respective doses of felodipine loaded nanoparticles were freshly prepared and administered by oral gavaged in a single dose. Acute toxicity was measured by mortality and survival time and also by clinical picture of intoxication and behavioural reactions. Animals on study were observed for any adverse reaction, like changes of body weight, condition of eye, nose and motor activity. On completion of the treatment, the animals were bled via the retro orbital plexus and sacrificed by cervical dislocation and necropsied to facilitate gross pathological examination of organs, viz. size and appearance of heart, lungs, liver, spleen and kidney [22].

\subsubsection{Histopathological studies}

The animals were sacrificed at the end of the 14 days and the organic tissues of heart, liver, spleen and kidneys were collected and fixed in 10\% formalin then subjected to histopathological examination. The tissues of organ samples were embedded in paraffin blocks, then sliced and placed onto glass slides. After histological staining the slides were observed and photos were taken using topical microscope and histopathological examination performed [23].

\subsubsection{Biochemical assay}

The blood samples were collected at 14th day and centrifuged at $4000 \mathrm{rpm}$ for $5 \mathrm{~min}$. The serum was kept at $-20^{\circ} \mathrm{C}$ until analyzed. The levels of serum glutamate oxaloacetic transaminase (SGOT), serum alkaline phosphate (SAP), serum glutamic pyruvic transaminase (SGPT), serum creatinine, serum bilirubin and proteins were analyzed with automatic analytical instrument (Hitachi, Japan) [24,25].

\subsection{Statistical analysis}

All values are expressed as mean \pm SD (standard deviation). Significant statistical differences were analyzed by one-way analysis of variance (ANOVA). In all comparisons, the difference was considered to be statistically significant at $\mathrm{p}<0.05{ }^{(*)}$.

\section{Results}

\subsection{Particle size and Zeta potential ( ()}

The mean particle sizes of the drug loaded PLGA nanoparticles were measured by Photon Correlation Spectroscopy (PCS). The sizes of the nanoparticles prepared with three different ratios of drug and polymer (1:2, 1:3 and 1:4) were in the nano range. The size distributions were monodispersed $(0.216$ to 0.442$)$ in all the nanoparticle formulations (Figure 1). No remarkable differences were observed between the sizes of nanoparticles obtained with different ratio of drug and polymer (Table 1). The zeta potential of the drug loaded nanoparticles exhibited negative values [26].

\subsection{Surface morphology studies of nanoparticles}

The surface morphology of the nanoparticles was measured using scanning electron microscopy. The shape of the particles as measured by SEM analysis revealed almost spherical in shape with relative smooth surface for all the formulations (Figure 2a). The AFM investigations showed the disc like shape of the particles (Figure 2b). The particle sizes obtained by SEM study were relatively smaller than that of the particle sizes obtained by Zetasizer [27].

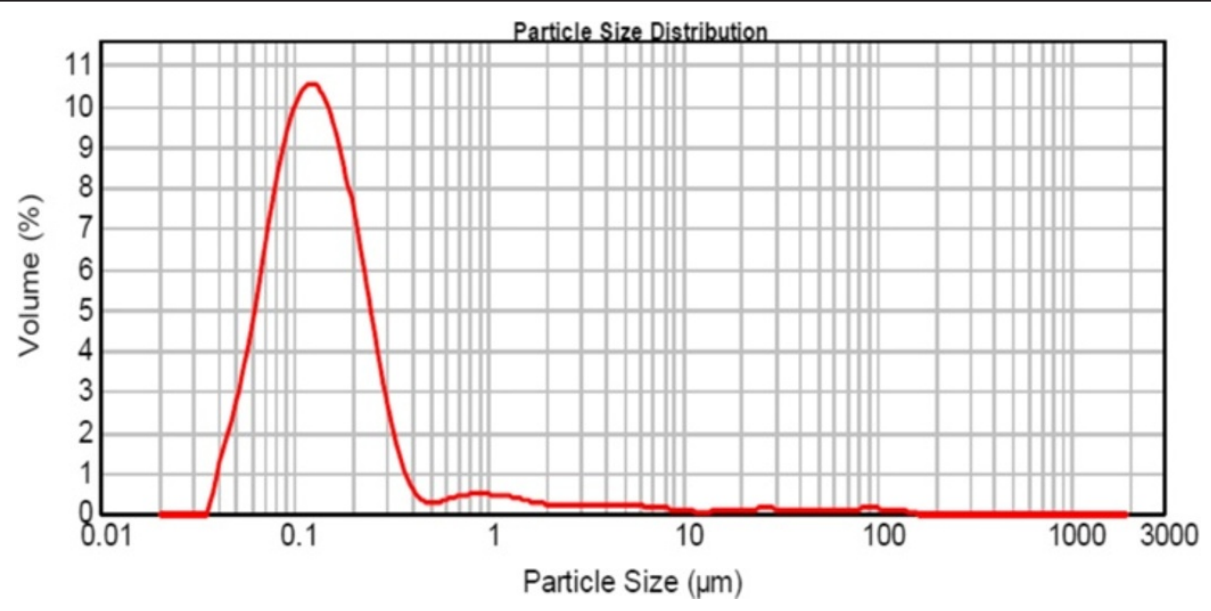

Figure 1 Mean particle size distributions of the felodipine loaded nanoparticles (F2) measured by Dynamic laser light scattering (data as mean \pm S.D; $n=6$ ). 
Table 1 Particle size, polydispersity index, drug loading and entrapment efficiency of three batches of felodipine loaded nanoparticles (Data represents mean \pm SD)

\begin{tabular}{llllll}
\hline Batch & Drug-polymer ratio & Particle size $(\mathbf{n m}) \pm$ SD $(\mathbf{n}=\mathbf{3})$ & Polydispersity index & Drug loading $(\% \mathbf{w} / \mathbf{w})$ & Entrapment Efficiency $(\% \mathbf{w} / \mathbf{w})$ \\
\hline F1 & $1: 2$ & $307 \pm 4.51$ & $0.442 \pm 0.098$ & $12.43 \pm 0.89$ & $68.45 \pm 0.869$ \\
F2 & $1: 3$ & $226 \pm 5.39$ & $0.216 \pm 0.009$ & $9.47 \pm 0.12$ & $85.28 \pm 0.328$ \\
F3 & $1: 4$ & $273 \pm 6.28$ & $0.254 \pm 0.004$ & $8.83 \pm 0.04$ & $83.23 \pm 0.365$ \\
\hline
\end{tabular}

EE $\left(\%^{w} / w\right)=\frac{\text { Weight of the drug in nanoparticle }}{\text { Weight of the polymer and drug added }} \times 100$.

$D L(\% w / w)=\frac{\text { Weight of the drug in nanoparticle }}{\text { Weight of the polymer and drug added }} \times 100$

\subsection{Drug entrapment efficiency and drug loading}

The entrapment efficiency and drug loading of the nanoparticles depend on the polymer, drug, surfactant etc. The entrapment efficiency becomes high when both drug and polymer shows more affinity to the same solvent. On the other hand the low entrapment is due to the high affinity of drug and polymer to the different solvents. Drug loading and entrapment efficiency were affected by the ratio of drug and polymer used in the formulation [28]. Therefore, we prepared three different formulations of the felodipine loaded nanoparticles taking three different ratios of drug- polymer. The nanoparticle formulation (F2) having drug- polymer ratio of 1:3, shows the high entrapment efficiency of $85.28 \%$ than the other two ratio $1: 2(68.45 \%)$ and $1: 4(83.23 \%)$ (Table 1$)$.

\subsection{Fourier transform infrared spectroscopy (FTIR)}

The FTIR spectral data were used to confirm chemical stability of felodipine in polymeric nanoparticles. FTIR spectra of pure felodipine, polymer and felodipine loaded nanoparticles are shown in (Figure 3). The interaction study between drug and polymer was evaluated. The absence of distinctive spectra in the felodipine loaded nanoparticles indicates that felodipine and PLGA was not involved in intermolecular interaction. However, the intensity of the peak at $3422 \mathrm{~cm}^{-1}, 2998 \mathrm{~cm}^{-1}$ and $2950 \mathrm{~cm}^{-1}$ were slightly decreased in the FTIR spectrum of the felodipine nanoparticles [29].

\subsection{Differential scanning calorimetry}

The physiochemical interaction of the drug encapsulated and the polymer was analyzed by differential scanning calorimetry. The study was carried out for the pure felodipine, polymer and felodipine loaded nanoparticles (Figure 4). DSC shows the characteristic peak for different compounds. The sharp endothermic peak at $\sim 144^{\circ} \mathrm{C}$ represents the melting point and crystalline anhydrous nature of native felodipine [30].

\subsection{In vitro drug release study}

The drug-polymer composition influences the in vitro drug release rate from the nanoparticles. The felodipine loaded nanoparticles shows biphasic drug release pattern, as the drug encapsulated in the polymeric nanoparticles and some amount of drug adsorb on the surface due to electrostatic interaction [31]. The in vitro drug release

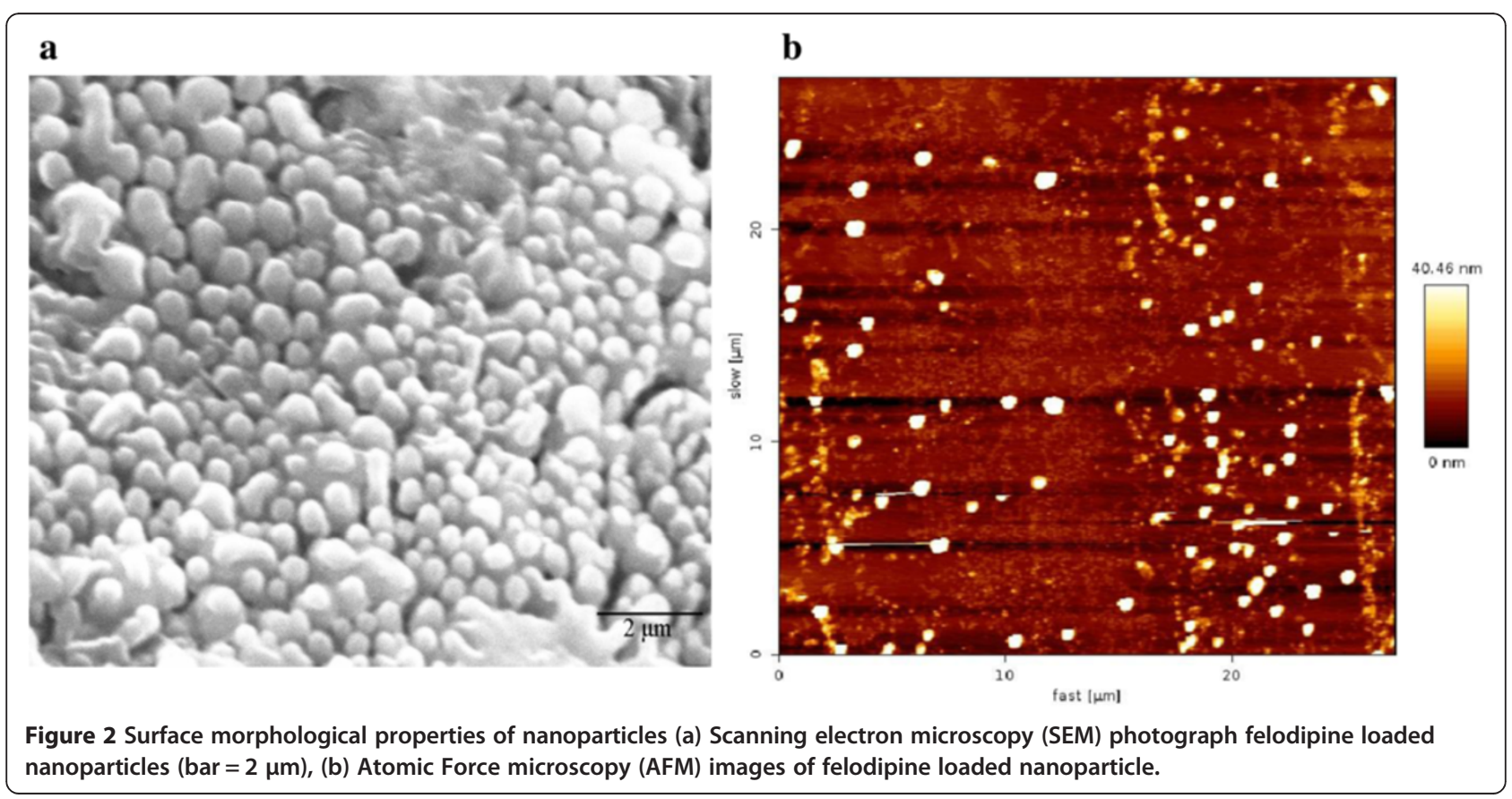




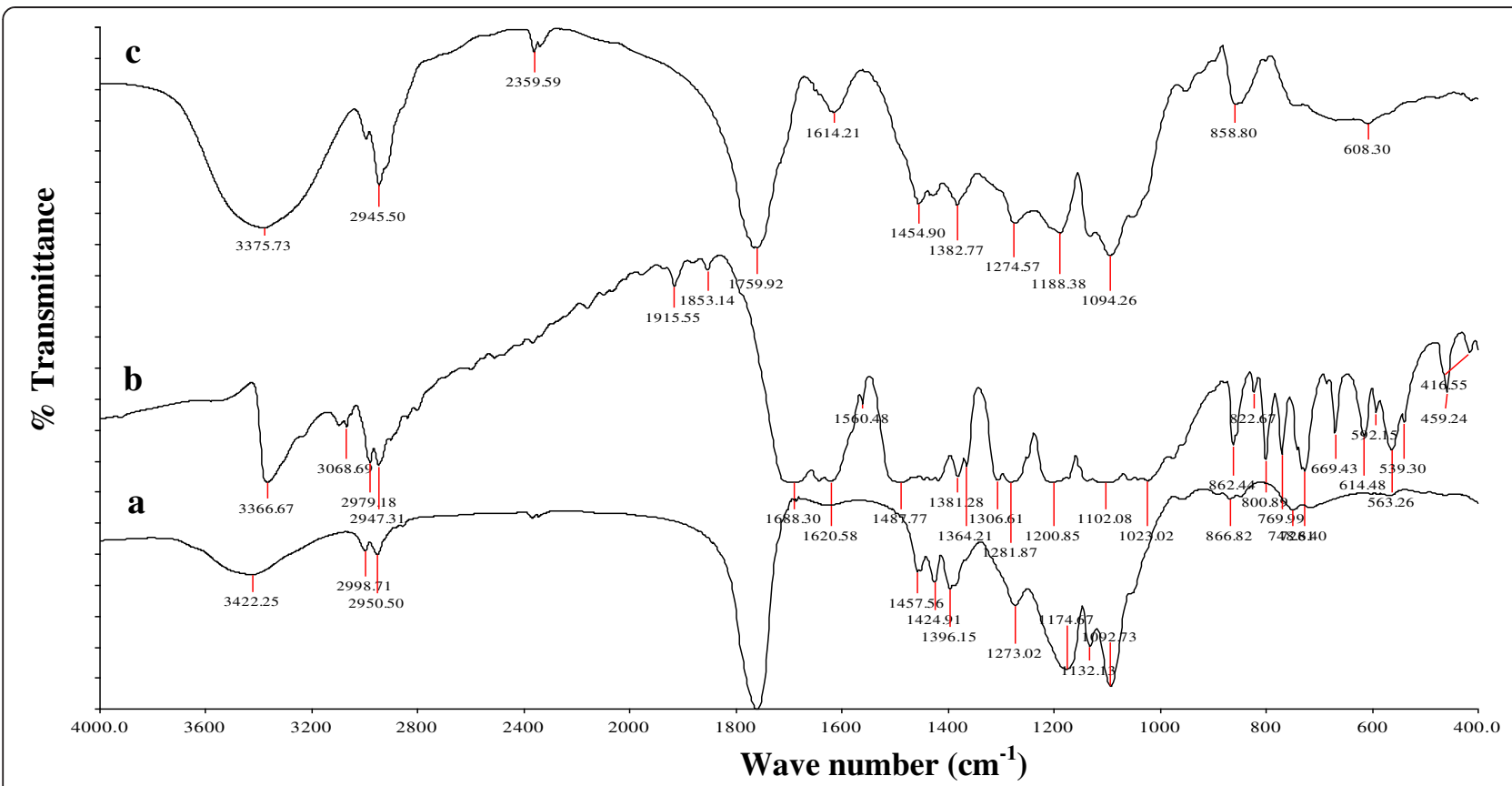

Figure 3 FTIR spectra of (a) Native felodipine, (b) PLGA polymer, (c) Felodipine loaded nanoparticles.

profile from prepared nanoparticles, intact drug powder and marketed formulation are shown in Figure 5. The felodipine loaded nanoparticles show slower drug release in comparison with intact drug powder and marketed formulation. The initial burst release of about $45 \%$ was observed for felodipine intact powder. This phenomenon was normal in the initial stage of many drug release systems [32] and it was reduced to $18 \%$ for felodipine loaded nanoparticles and also exhibited slow drug release at least $96 \mathrm{~h}$ due to the coating of polymer around the drug.

The drug release data were fitted with various kinetic models, data not shown. The drug release kinetics of the formulation F2 was pre-eminently explained by zero

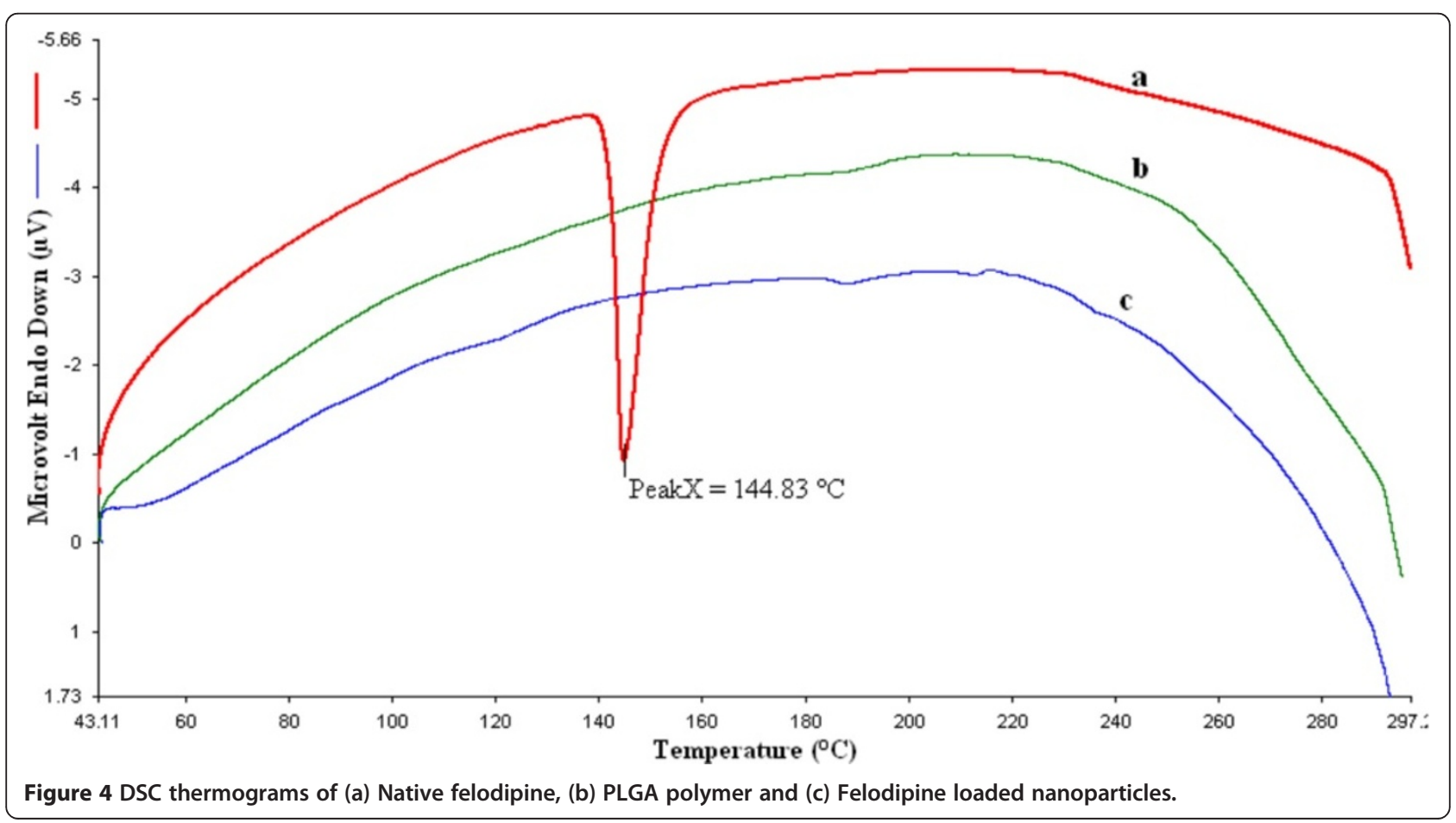




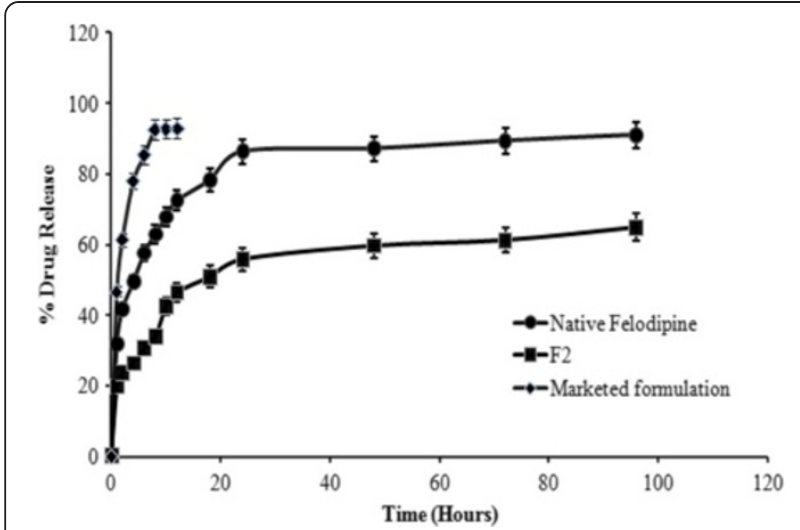

Figure $\mathbf{5}$ In vitro drug release study of felodipine loaded nanoparticles, felodipine intact powder and marketed formulation respectively. Values represent mean \pm S.D. $(n=3)$.

order and first order kinetic, followed by Higuchi model, as it showed the highest linearity. From the Peppas model the ' $n$ ' value (0.284) was calculated from the slope and found to be less than 0.5 , indicating that the release mechanism from the nanoparticles was diffusion controlled.

\subsection{Toxicity studies}

The felodipine loaded nanoparticles can cross the small intestinal wall by persorption and distributed in various parts of the body [33]. The animals in various treatment groups as well as control group did not showed any mortality throughout the observation period except group $\mathrm{V}$. In the treatment group $\mathrm{V}$ all the animals were died within 12 hours after the administration of the dose (480 $\mathrm{mg} / \mathrm{kg}$ body weight). Throughout the observation period, the animals in group I, II, III and IV did not show any abnormality or behavioural changes (Table 2). Necroscopic examination revealed no gross changes in the vital organs of the animals in the treatment group I, II, III and IV (Figure 6).

The pathological examinations of different organs of wistar albino mice treated with $240 \mathrm{mg} / \mathrm{kg}$ body weight of felodipine loaded nanoparticles were carried out.

Table 2 Behavioral parameters of drug loaded PLGA nanoparticles treated albino mice

\begin{tabular}{ll}
\hline Parameters & Results \\
\hline Motor activity & Normal \\
Respiration & Normal \\
Salivation & Normal \\
Convulsions & Negative \\
Body weight & No significant change \\
Skin colour & Normal \\
Righting reflex & Positive \\
\hline
\end{tabular}

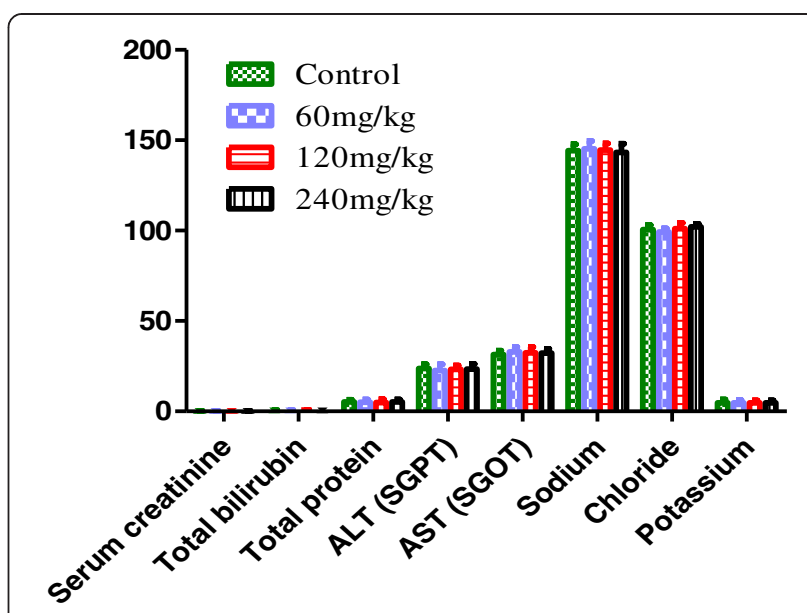

Figure 6 Biochemical parameters in control and nanoparticle treated group (I, II, III and IV) in wistar albino mice $\left({ }^{*} \mathrm{p}<0.05\right)$.

There was no change in histopathology of glomerulus and tubules of kidney. Liver showed normal hepatocytes and absence of necrosis as compared to control group. The pathological examinations revealed that heart, kidney, liver and lungs were not exposed to nanotoxicity after oral administration of the felodipine loaded nanoparticles when compared with control groups (Figure 7).

\section{Discussion}

The felodipine loaded PLGA nanoparticles were prepared by single emulsion solvent evaporation method. The particle sizes of the drug loaded nanoparticles are important parameter of the nanoparticles, which has direct impact on drug release, cellular uptake, biodistribution and stability. The drug loaded nanoparticle shows negative zeta potential and the result suggest the external localization of free drugs, adsorbed on the surface of the polymeric nanoparticles [34]. These higher negative zeta potential values reduce the particle aggregation and smaller particle size may facilitate the effective absorption of the drug loaded nanoparticles in the biological systems. The surface morphology of the nanoparticles was studied by SEM and AFM. The electron microscope allows the visualization of the nanoparticle surface, whereas zetasizer measures the particle sizes surrounded by hydrodynamic layer, so it provides larger size than the SEM images [26]. Drug loading and entrapment efficiency were measured by RP-HPLC method and the results show increased entrapment efficiency in F2 formulation having drugpolymer ratio of 1:3 [28]. The drug- polymer ratio was taken into consideration to improve the entrapment efficiency of the nanoparticle systems keeping the other parameters constant, and the result showed that, the increase in the drug-polymer ratio up to $1: 4$, the entrapment efficiency was almost remained constant. This may be due to the saturation level of felodipine inside the 


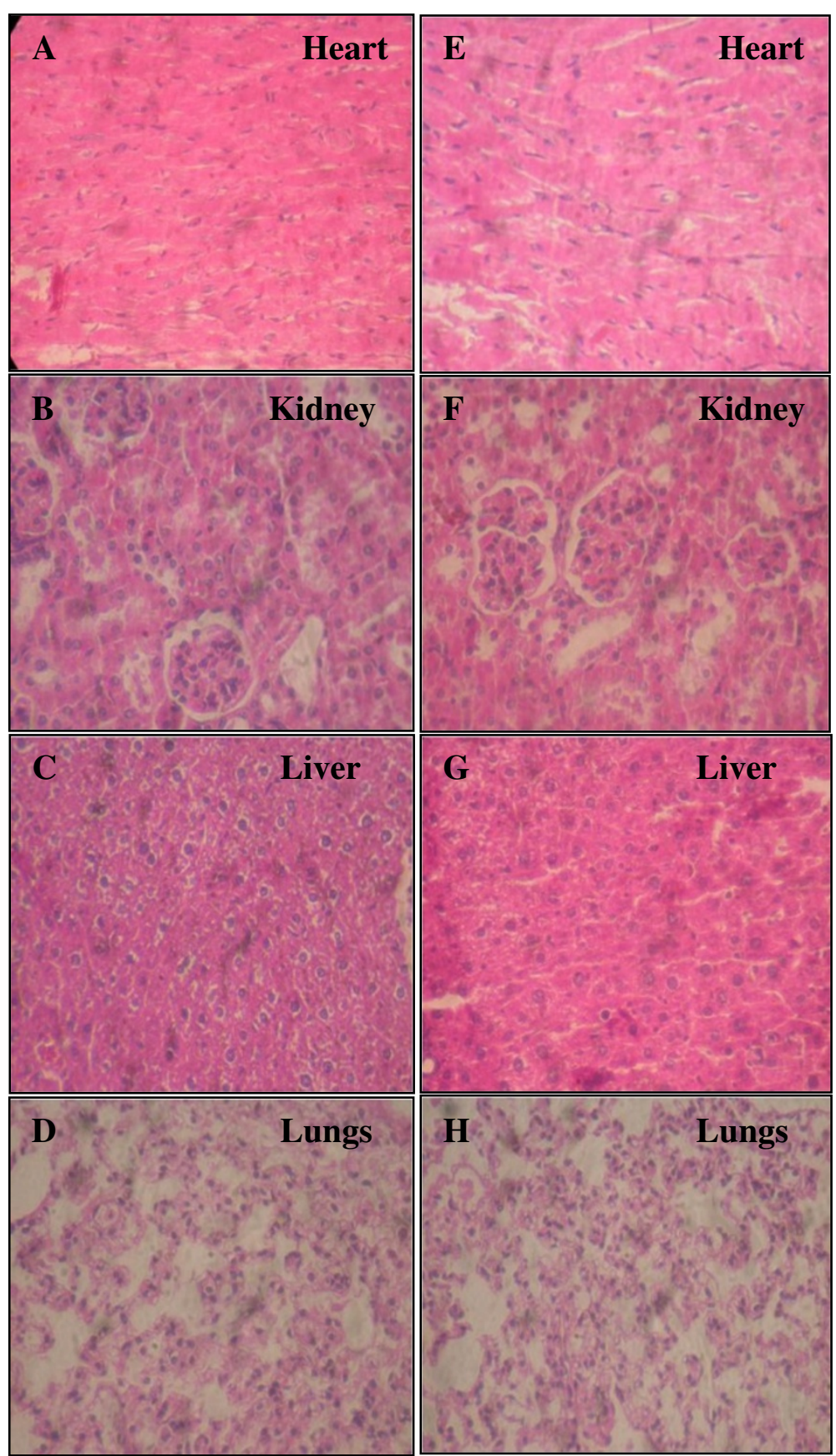

Figure 7 The histopathological slides of wistar albino mice treated with $240 \mathrm{mg} / \mathrm{kg}$ body weight of felodipine loaded nanoparticles formulation compared with control. (A-D) Slide shows the heart, kidney, liver and lungs of control mice. (E-H) Slide shows the heart, kidney, liver and lungs of felodipine nanoparticle treated mice.

nanoparticles. In the case of drug loading, as it increases a more porous polymeric structure forms, with large number of channels and hollow spaces. The drug can easily escape to the outer phase and decrease the drug inside the polymeric matrix of the nanoparticles [29]. Hence, the nanoparticle formulation (F2) having drugpolymer ratio of $1: 3$ with highest entrapment efficiency and less particle size was selected and used for the further studies. The FTIR spectrum of the felodipine nanoparticles indicates the chemical stability of the felodipine inside the nanoparticles due to the intermolecular hydrogen bonding between drug and polymer [29]. The drug loaded nanoparticles depicted no distinctive peak of the felodipine in the DSC profiles owing to the decreased 
crystallinity in the formulations and/or drug solvation in the amorphous carrier as well as solid state interaction induced by heating. The in vitro drug release signifies that the prepared nanoparticulate system possess sustained release properties. The initial burst release from the nanoparticles was due to surface adsorbed drug. After $8 \mathrm{~h}$ the rapid drug release was identified and this could be as a result of surface adsorbed drug released due to polymer erosion. It suggests that the combination of dissolution, diffusion and erosion are the possible mechanism to release the drug from the nanoparticles. The release kinetics of felodipine from the nanoparticles was preeminently explained by zero order and first order kinetic and it shows release of the drug was by diffusion mechanism [32]. To further explain the in vivo toxicity of the felodipine loaded nanoparticles, the wistar albino mice were treated with various concentration of drug loaded nanoparticles and it was found that no abnormality or behavioural changes in the group I, II, III and IV. The necroscopic examination revealed no gross changes occurred in the vital organs of the animals after the treatment. Similarly, the pathological examination of the different organ of the treated animal shows no change in histopathology. Thus the felodipine loaded nanoparticles were found to be orally safe at the single limit dose equivalent to $240 \mathrm{mg} / \mathrm{kg}$ body weight of felodipine.

\section{Conclusions}

Felodipine loaded nanoparticles were prepared successfully by applying emulsion-solvent evaporation technique. The nanoparticles were formulated in reproducible methodology and were able to improve the physicochemical characteristics of the native drug. The DSC study confirmed that there was no intermolecular interaction between the felodipine and PLGA. The prepared drug loaded nanoparticles showed slow release of the drug with the reduced burst release in comparison with intact drug powder. The various toxicity studies indicated the oral safety and the cellular safety of the developed felodipine loaded nanoparticles. These results may prove to be beneficial for the prolonged utilization of the formulation as an adjuvant anti-hypertensive therapy. Thus, the felodipine loaded nanoparticles may provide an effective drug delivery system for the hypertension therapy.

\section{Competing interests}

The authors declare that they have no competing interests.

\section{Authors' contribution}

UJ synthesized and chacterised the drug loaded nanoparticles, participate in animal experiments and drafted the manuscript. AKM helped in performing the characterization of nanoparticles and drafted the manuscript. SLP helps in animal experiment and biochemical parameter estimation. PKM and GPM scrutinized the results, helped in histo-pathology slide preparations and final cheek up of drafted manuscript. All authors read and approved the final manuscript.

\section{Acknowledgments}

Utpal Jana is grateful to University Grants Commission (UGC), Government of India, for providing UGC-BSR fellowship. All the authors are deeply indebted to Dr. K. Kanann, Professor and Head, Department of Pharmacy, Annamalai University and Dr. R. Manavalan, UGC-Basic Science Research Faculty Fellow (UGC-BFF), Govt. of India, for kind support.

Received: 24 July 2014 Accepted: 18 September 2014

Published online: 11 December 2014

\section{References}

1. M Ezzati, S Vander Hoorn, CM Lawes, R Leach, WP James, AD Lopez, A Rodgers, CJ Murray, Rethinking the "diseases of affluence" paradigm: global patterns of nutritional risks in relation to economic development. PLoS Med. 2, e133-e2005 (2005)

2. MJ Brown, Science, medicine, and the future. Hypertension. Bmj. 314, 1258-1261 (1997)

3. G Mancia, R Sega, C Milesi, G Cesana, A Zanchetti, Blood-pressure control in the hypertensive population. Lancet. 349, 454-457 (1997)

4. JA Staessen, JG Wang, L Thijs, Cardiovascular prevention and blood pressure reduction: a quantitative overview updated until 1 March 2003. J. Hypertens. 21, 1055-1076 (2003)

5. MJ Lawrence, GD Rees, Microemulsion-based media as novel drug delivery systems. Adv. Drug Deliv. Rev. 45, 89-121 (2000)

6. VJ Stella, RA Rajewski, Cyclodextrins: their future in drug formulation and delivery. Pharm. Res. 14, 556-567 (1997)

7. B Sjostrom, B Bergenstahl, B Kronberg, A method for the preparation of submicron particles of sparingly water-soluble drugs by precipitation in oil-in-water emulsions. II: influence of the emulsifier, the solvent, and the drug substance. J. Pharm. Sci. 82, 584-589 (1993)

8. I Brigger, C Dubernet, P Couvreur, Nanoparticles in cancer therapy and diagnosis. Adv. Drug Deliv. Rev. 54, 631-651 (2002)

9. C Vauthier, C Dubernet, C Chauvierre, I Brigger, P Couvreur, Drug delivery to resistant tumors: the potential of poly(alkyl cyanoacrylate) nanoparticles. J. Control. Release. 93, 151-160 (2003)

10. VC Furtado, P Legrand, A Gulik, O Bourdon, R Gref, D Labarre, G Barratt, Relationship between complement activation, cellular uptake and surface physicochemical aspects of novel PEG-modified nanocapsules. Biomaterials. 22, 2967-2979 (2001)

11. AF Ourique, AR Pohlmann, SS Guterres, RC Beck, Tretinoin-loaded nanocapsules: preparation, physicochemical characterization, and photostability study. Int. J. Pharm. 352, 1-4 (2008)

12. C Jacobs, O Kayser, RH Muller, Production and characterisation of mucoadhesive nanosuspensions for the formulation of bupravaquone. Int. J. Pharm. 214, 3-7 (2001)

13. F Debuigne, J Cuisenaire, L Jeunieau, B Masereel, JB Nagy, Synthesis of nimesulide nanoparticles in the Microemulsion epikuron/isopropyl myristate/ water/n-butanol (or isopropanol). J. Colloid. Interface Sci. 243, 90-101 (2001)

14. M Trotta, M Gallarate, F Pattarino, S Morel, Emulsions containing partially water-miscible solvents for the preparation of drug nanosuspensions. J. Control. Release. 76, 119-128 (2001)

15. K Adibkia, MR Siahi Shadbad, A Nokhodchi, A Javadzedeh, M Barzegar-Jalali, J Barar, G Mohammadi, Y Omidi, Piroxicam nanoparticles for ocular delivery: physicochemical characterization and implementation in endotoxin-induced uveitis. J. Drug Target. 15, 407-416 (2007)

16. R Pignatello, C Bucolo, P Ferrara, A Maltese, A Puleo, G Puglisi, Eudragit RS100 nanosuspensions for the ophthalmic controlled delivery of ibuprofen. Eur. J. Pharm. Sci. 16, 53-61 (2002)

17. RA Jain, The manufacturing techniques of various drug loaded biodegradable poly(lactide-co-glycolide) (PLGA) devices. Biomaterials. 21, 2475-2490 (2000)

18. AK Mohanty, F Dilnawaz, C Mohanty, SK Sahoo, Etoposide-loaded biodegradable amphiphilic methoxy (poly ethylene glycol) and poly (epsilon caprolactone) copolymeric micelles as drug delivery vehicle for cancer therapy. Drug Deliv. 17, 330-342 (2010)

19. A Trapani, J Sitterberg, U Bakowsky, T Kissel, The potential of glycol chitosan nanoparticles as carrier for low water soluble drugs. Int. J. Pharm. 375, 97-106 (2009)

20. PV Devarajan, GS Sonavane, Preparation and in vitro/in vivo evaluation of gliclazide loaded Eudragit nanoparticles as a sustained release carriers. Drug Dev. Ind. Pharm. 33, 101-111 (2007) 
21. S Jain, S Saraf, Influence of processing variables and in vitro characterization of glipizide loaded biodegradable nanoparticles. Diabetes and Metabolic Myndrome. Clinical. Res. Rev. 3, 113-117 (2009)

22. SE Gelperina, AS Khalansky, IN Skidan, ZS Smirnova, Al Bobruskin, SE Severin, B Turowski, FE Zanella, J Kreuter, Toxicological studies of doxorubicin bound to polysorbate 80-coated poly(butyl cyanoacrylate) nanoparticles in healthy rats and rats with intracranial glioblastoma. Toxicol Lett. 126, 131-141 (2002)

23. UM Dhana Lekshmi, G Poovi, N Kishore, PN Reddy, In vitro characterization and invivo toxicity study of repaglinide loaded poly (methyl methacrylate) nanoparticles. Int J Pharm 396, 194-203 (2010)

24. CW Lam, JT James, R McCluskey, RL Hunter, Pulmonary toxicity of single-wall carbon nanotubes in mice 7 and 90 days after intratracheal instillation. Toxicol Sci. 77, 126-134 (2004)

25. G Oberdorster, E Oberdorster, J Oberdorster, Nanotoxicology: an emerging discipline evolving from studies of ultrafine particles. Environ Health Perspect. 113, 823-839 (2005)

26. U Jana, AK Mohanty, PK Manna, GP Mohanta, Preparation and characterization of nebivolol nanoparticles using Eudragit(R) RS100. Colloids Surf B Biointerfaces. 113, 269-275 (2014)

27. U Jana, AK Mohanty, SL Pal, PK Manna, GP Mohanta, Preparation and in vitro characterisation of felodipine loaded Eudragit ${ }^{\oplus}$ RS100 nanoparticles. Int. J. Pharm. Pharm. Sci. 6, 564-567 (2014)

28. P Dongming, K Huang, Y Liu, S Lin, Preparation of novel polymeric microspheres for controlled release of finasteride. Int. J. Pharm. 342, 82-86 (2007)

29. C Witschi, E Doelker, Influence of the microencapsulation method and peptide loading on poly(lactic acid) and poly(lactic-co-glycolic acid) degradation during in vitro testing. J. Control. Release. 51, 327-341 (1998)

30. AR Tapas, PS Kawtikwar, DM Sakarkar, Enhanced dissolution rate of felodipine using spherical agglomeration with Inutec SP1 by quasi emulsion solvent diffusion method. Res Pharm Sci. 4, 77-84 (2009)

31. SJ Douglas, SS Davis, L Illum, Nanoparticles in drug delivery. Crit. Rev. Ther. Drug Carrier Syst. 3, 233-261 (1987)

32. E Leo, F Forni, MT Bernabei, Surface drug removal from ibuprofen-loaded PLA microspheres. Int. J. Pharm. 196, 1-9 (2000)

33. JF Hillyer, RM Albrecht, Gastrointestinal persorption and tissue distribution of differently sized colloidal gold nanoparticles. J Pharm Sci. 90, 1927-1936 (2001)

34. K Dillen, J Vandervoort, G Van den Mooter, A Ludwig, Evaluation of ciprofloxacin-loaded Eudragit RS100 or RL100/PLGA nanoparticles. Int. J. Pharm. 314, 72-82 (2006)

doi:10.1186/s40580-014-0031-5

Cite this article as: Jana et al.: Felodipine loaded PLGA nanoparticles: preparation, physicochemical characterization and in vivo toxicity study. Nano Convergence 2014 1:31.

\section{Submit your manuscript to a SpringerOpen ${ }^{\circ}$ journal and benefit from:}

- Convenient online submission

- Rigorous peer review

- Immediate publication on acceptance

- Open access: articles freely available online

- High visibility within the field

- Retaining the copyright to your article

Submit your next manuscript at $\gg$ springeropen.com 